\title{
EXIT PROBLEMS FOR SPECTRALLY \\ NEGATIVE LÉVY PROCESSES REFLECTED \\ AT EITHER THE SUPREMUM OR THE INFIMUM
}

\author{
XIAOWEN ZHOU, ${ }^{*}$ Concordia University
}

\begin{abstract}
For a spectrally negative Lévy process $X$ on the real line, let $S$ denote its supremum process and let $I$ denote its infimum process. For $a>0$, let $\tau(a)$ and $\kappa(a)$ denote the times when the reflected processes $\hat{Y}:=S-X$ and $Y:=X-I$ first exit level $a$, respectively; let $\tau_{-}(a)$ and $\kappa_{-}(a)$ denote the times when $X$ first reaches $S_{\tau(a)}$ and $I_{\kappa(a)}$, respectively. The main results of this paper concern the distributions of $\left(\tau(a), S_{\tau(a)}, \tau_{-}(a), \hat{Y}_{\tau(a)}\right)$ and of $\left(\kappa(a), I_{\kappa(a)}, \kappa_{-}(a)\right)$. They generalize some recent results on spectrally negative Lévy processes. Our approach relies on results concerning the solution to the two-sided exit problem for $X$. Such an approach is also adapted to study the excursions for the reflected processes. More explicit expressions are obtained when $X$ is either a Brownian motion with drift or a completely asymmetric stable process.
\end{abstract}

Keywords: Spectrally negative Lévy process; reflected Lévy process; fluctuation theory; exit problem; excursion; risk model; ruin time

2000 Mathematics Subject Classification: Primary 60G51

Secondary 60B 15

\section{Introduction and preliminaries}

Let $X$ be an $\mathbb{R}$-valued spectrally negative Lévy process, i.e. a Lévy process with no positive jumps. Write $\mathrm{P}^{x}$ for the probability law of $X$ when $X_{0}=x$. Write $\mathrm{P}:=\mathrm{P}^{0}$. Then there exists a $\sigma$-finite Lévy measure $\Pi$ on $(-\infty, 0)$ such that

$$
\int_{-\infty}^{0} 1 \wedge x^{2} \Pi(\mathrm{d} x)<\infty
$$

and

$$
\mathrm{E}\left[\exp \left(\lambda X_{t}\right)\right]=\mathrm{e}^{t \psi(\lambda)}, \quad \lambda \geq 0,
$$

where the Laplace exponent $\psi$ is of the form

$$
\psi(\lambda)=m \lambda+\frac{\sigma^{2} \lambda^{2}}{2}+\int_{-\infty}^{0}\left(\mathrm{e}^{\lambda x}-1-\lambda x \mathbf{1}_{\{x>-1\}}\right) \Pi(\mathrm{d} x) .
$$

We always assume that neither $X$ nor $-X$ is a subordinator.

Set

$$
S_{t}:=\sup _{0 \leq s \leq t} X_{s} \vee 0 \quad \text { and } \quad I_{t}:=\inf _{0 \leq s \leq t} X_{s} \wedge 0
$$

Received 27 October 2006; revision received 12 September 2007.

* Postal address: Department of Mathematics and Statistics, Concordia University, 1455 de Maisonneuve Blvd. W., Montreal, Quebec, H3G 1M8 Canada. Email address: zhou@alcor.concordia.ca

Supported by an NSERC operating grant. 
for the supremum process and the infimum process of $X$, respectively. Then $\hat{Y}:=S-X$ is the process reflected at the supremum, and $Y:=X-I$ is the process reflected at the infimum. Both $Y$ and $\hat{Y}$ are known to be Feller processes, and $S$ is known to be a local time at 0 for $\hat{Y}$. We refer the reader to [3, Chapters VI and VII], [6], [8, Chapters 4 and 9], and [13, Chapter 8] for introductions on the reflected Lévy processes.

Reflected Lévy processes arise naturally in storage and queueing theory. They are used to define the dam-content process for a dam and the virtual waiting-time process for an M/G/1 queue; see [6] for some results and references. A two-sided reflected Brownian motion is used in [11] to model the inventory process in a stochastic flow system. A reflected Lévy process can also be used to describe the heavy-traffic limit of the queue-content process in a first-in-first-out queue with a finite capacity; see [24] for a detailed review.

Reflected Lévy processes find extensive applications in risk theory for insurance, where the surplus process is often defined via a Lévy process with no positive jumps, and the reflected process describes a model with a constant dividend barrier. We refer the reader to [9] for discussions and the literature therein. See [1] for an application of the reflected Lévy process in mathematical finance.

Reflected spectrally positive Lévy processes are also used in [16] to study the genealogical structure of a continuous-state branching process which undergoes Lévy branching. They are used to define the height processes for the Lévy trees.

Other earlier works on reflected Lévy processes can be found in [2], [10], and [22].

For $a>0$, let

$$
\begin{gathered}
\tau(a):=\inf \left\{t \geq 0: \hat{Y}_{t} \geq a\right\}, \\
\tau_{-}(a):=\sup \left\{t \geq 0: t \leq \tau(a), \hat{Y}_{t}=0\right\}, \\
\kappa(a):=\inf \left\{t \geq 0: Y_{t} \geq a\right\},
\end{gathered}
$$

and

$$
\kappa_{-}(a):=\sup \left\{t \geq 0: t \leq \kappa(a), Y_{t}=0\right\},
$$

with the conventions inf $\varnothing=\infty$ and $\sup \varnothing=0$.

The joint distribution of $\left(\tau(a), S_{\tau(a)}, \hat{Y}_{\tau(a)}\right)$ is of interest in both queuing theory and the risk models for insurance; see [9] and [25], and the literature therein for more details. More specifically, in the risk model with a dividend barrier, $\tau(a)$ is related to the ruin time, $S_{\tau(a)}$ is related to the total amount of dividend payments until ruin, $\tau_{-}(a)$ is related to the last time when dividend is paid before ruin, and $\hat{Y}_{\tau(a)}-a$ is related to the severity of ruin. To the author's knowledge such a distribution (in terms of its Laplace transform) was first obtained in [23] for a Brownian motion with drift. We also refer the reader to [21, Exercise VI.4.9] and [25], where the Laplace transform is recovered by Itô's formula and the optimal stopping theorem applied to a cleverly chosen martingale defined via $\hat{Y}$ and $S$.

In recent years there has been renewed interest in the study of reflected spectrally negative Lévy processes; see [1], [7], [12], [14], [17], [18], [19], and [20]. In all these papers the exit problems are considered for the reflected processes. We are going to elaborate these works further in the following.

When $X$ is a spectrally negative Lévy process, the exit problem for $\hat{Y}$ is considered and the joint Laplace transform for $\left(\tau(a), \hat{Y}_{\tau(a)}\right)$ is obtained in [1], where Itô-excursion theory is used in the proof. This result is extended in [19] to one concerning $\left(\tau(a), S_{\tau(a)}, \hat{Y}_{\tau(a)-}, \Delta X_{\tau(a)}\right)$. Similar problems are also discussed in [17] by introducing $\hat{Y}$-related martingales. The Laplace 
transform for $\kappa(a)$ is obtained in [18], where a martingale argument is employed. More computations on the reflected processes using the excursion theory can be found in [7]. The distribution for $\int_{0}^{\tau(a)} \mathrm{e}^{-\delta t} \mathrm{~d} S_{t}$, which can be interpreted in risk models as the present value of the total amount of dividends before ruin, is studied in both [14] and [20] using different approaches. For strictly stable processes $X$ with possibly two-sided jumps, an explicit expression for the distribution of $\hat{Y}_{\tau(a)}$ can be found in [12].

In this paper we continue to implement the fluctuation theory for Lévy processes to study the abovementioned joint distribution. A key observation is that the exit problem for the reflected process is, at its heart, a two-sided exit problem for the underlying Lévy process. More precisely, by first considering the exit time of process $X$ from the interval $(-a, \varepsilon)$ and then letting $\varepsilon \rightarrow 0+$, in Theorem 4.1 we recover the multidimensional Laplace transform for $\left(\tau(a), S_{\tau(a)}, \tau_{-}(a), \hat{Y}_{\tau(a)}\right)$. To this end, the solution to the so-called two-sided exit problem in [5], which we will introduce in Section 2, plays a key role in this work. Similarly, by considering how process $X$ exits the interval $(-\varepsilon, a)$ we also obtain a Laplace transform for $\left(\kappa(a), I_{\kappa(a)}, \kappa_{-}(a)\right)$ in Theorem 5.1. Our approach is a direct application of the solution to the two-sided exit problem together with the strong Markov property. Therefore, it has the advantage of being self contained. In the other literature, martingale methods, the WienerHopf factorization, or Itô's excursion theory are often exploited.

We remark that our approach has the spirit of Chaumont and Doney's characterization of the excursion measure via the limit of the probability law of the killed Lévy process normed by the renewal function for the appropriate ladder height process; see [8, Proposition 17]. It is certainly evident that most of the results in this paper can also be recovered via Itô's excursion theory.

For $x \geq 0$, write

$$
L^{-1}(x):=\inf \left\{t \geq 0: S_{t}>x\right\}=\inf \left\{t \geq 0: X_{t}>x\right\} .
$$

The process $L^{-1}$ is the so-called inverse local time or the ladder height time of $\hat{Y}$. In Section 4 the same idea as that used in the proof of Theorem 4.1 leads to the defective distribution of $L^{-1}(x)$ when it is restricted to $[0, \tau(a))$. The approach in this paper is further exploited in Section 4 to find the joint distribution on the starting time, the height, and the duration of the first excursion for $\hat{Y}$ with height at least $a$.

As examples, the Brownian motion with drift and the completely asymmetric stable process are studied in detail in Section 5. Some explicit expressions are obtained.

Throughout this paper, our basic data are the Lévy measure $\Pi$ and the scale function $W^{(q)}$, which will be defined in Section 2.

\section{The two-sided exit problem for spectrally negative Lévy processes}

For $a, b>0$, throughout this section, we will always write

$$
T:=\inf \left\{t \geq 0: X_{t} \notin(-a, b)\right\} .
$$

The fluctuation theory for Lévy processes concerns the joint distribution of $T$ and $X_{T}$; see [6] for a review of earlier work. We refer the reader to [5], [8, Chapter 9], and [13, Chapter 8] for a more recent and comprehensive coverage.

In the following we collect some results that we will need later in the paper.

The Laplace exponent $\psi(x)$ is strictly convex for $x \geq 0$. Moreover, $\psi(0)=0$ and $\lim _{x \rightarrow \infty} \psi(x)=\infty$. Then, for $\lambda>0$, the equation $\psi(x)=\lambda$ has exactly one positive 
solution. In the sequel we denote the unique positive solution by $\Phi(\lambda)$, i.e. $\psi(\Phi(\lambda))=\lambda$. Set $\Phi(0):=\lim _{\lambda \rightarrow 0+} \Phi(\lambda)$.

For the so-called one-sided exit problem with $a=\infty$, it is known that [6, Theorem VII.1]

$$
\mathrm{E}\left[\mathrm{e}^{-q T}\right]=\mathrm{E}\left[\exp \left(-q L^{-1}(b)\right)\right]=\mathrm{e}^{-b \Phi(q)}, \quad q>0 .
$$

A solution to the two-sided exit problem, where $a \neq \infty$ and $b \neq \infty$, can be found in [5]. More precisely, in [5] it was pointed out that

$$
\mathrm{E}\left[\mathrm{e}^{-q T} \mathbf{1}_{\left\{X_{T}=b\right\}}\right]=\frac{W^{(q)}(a)}{W^{(q)}(a+b)}
$$

and ([5, Corollary 1], note that the two $q$ s should appear in its original statement)

$$
\begin{aligned}
\mathrm{E}\left[\mathrm{e}^{-q T}\right] & =\hat{W}^{(q)}(a, b) \\
& :=1+q \bar{W}^{(q)}(a)-\frac{q W^{(q)}(a) \bar{W}^{(q)}(a+b)}{W^{(q)}(a+b)},
\end{aligned}
$$

where the (generalized) scale function $W^{(q)}$ is a continuous function over $[0, \infty)$ which is determined by its Laplace transform

$$
\int_{0}^{\infty} \mathrm{e}^{-\lambda x} W^{(q)}(x) \mathrm{d} x=\frac{1}{\psi(\lambda)-q}, \quad \lambda>\Phi(q),
$$

and

$$
\bar{W}^{(q)}(x):=\int_{0}^{x} W^{(q)}(r) \mathrm{d} r, \quad x \geq 0 .
$$

We always write $W$ for $W^{(0)}$. By Equation (9) of [5] we have

$$
W^{(q)}(x)=\sum_{k=1}^{\infty} q^{k-1} W^{* k}(x),
$$

where ' $*$ ' stands for the convolution operator.

By Theorem 1 and Corollary 2 of [5], for $z<-a$, we have

$$
\mathrm{E}\left[\mathrm{e}^{-q T} \mathbf{1}_{\left\{X_{T} \in \mathrm{d} z\right\}}\right]=\int_{-a}^{b} \mathrm{~d} y \tilde{W}^{(q)}(a, b, y) \Pi(-y+\mathrm{d} z),
$$

where

$$
\tilde{W}^{(q)}(a, b, y):=\frac{W^{(q)}(a) W^{(q)}(b-y)}{W^{(q)}(a+b)}-W^{(q)}(-y) \mathbf{1}_{\{y \leq 0\}} .
$$

Therefore,

$$
\begin{aligned}
& \mathrm{E}\left[\mathrm{e}^{-q T} \mathbf{1}_{\left\{X_{T}=-a\right\}}\right] \\
& \quad=\mathrm{E}\left[\mathrm{e}^{-q T}\right]-\mathrm{E}\left[\mathrm{e}^{-q T} \mathbf{1}_{\left\{X_{T}=b\right\}}\right]-\int_{-\infty}^{-a-} \mathrm{E}\left[\mathrm{e}^{-q T} \mathbf{1}_{\left\{X_{T} \in \mathrm{d} z\right\}}\right] \\
& \quad=\hat{W}^{(q)}(a, b)-\frac{W^{(q)}(a)}{W^{(q)}(a+b)}-\int_{-a}^{b} \mathrm{~d} y \tilde{W}^{(q)}(a, b, y) \Pi((-\infty,-y-a)) .
\end{aligned}
$$


In the following we collect some known properties of $W^{(q)}$. It is clear, from (2.2), that the scale function $W^{(q)}(x)$ is strictly increasing for $x>0$ and for $q>0, W^{(q)}(x) \rightarrow \infty$ as $x \rightarrow \infty$. We have $W^{(q)}(0)=0$ if and only if the paths of $X$ are of unbounded variation, and also if and only if 0 is regular for $(-\infty, 0)$ for $X$ [3, Corollary VII.5]. It follows, from (2.5), that

$$
\lim _{x \rightarrow 0+} \frac{W^{(q)}(x)}{W(x)}=1 ;
$$

see [7, Lemma 1].

If the paths of $X$ are of unbounded variation then $W^{(q) \prime}(x)$, the derivative of $W^{(q)}(x)$, exists and is continuous for all $x>0$. If the paths of $X$ are of bounded variation then both $W_{+}^{(q) \prime}(x)$ and $W_{-}^{(q) \prime}(x)$, the right derivative and the left derivative of $W^{(q)}(x)$, respectively, exist and agree except for the set

$$
\mathscr{D}:=\{x>0: \Pi \text { has a positive mass at }-x\} ;
$$

see [7, Lemma 1]. Furthermore,

$$
W_{+}^{\prime}(0):=\lim _{x \rightarrow 0+} \frac{W(x)-W(0)}{x}
$$

is either a positive constant or $+\infty\left[18\right.$, Lemma 4]. Therefore, whenever $W^{(q)}(0)=0$, combining (2.8) and (2.9) we have $W(0)=0$ and

$$
W_{+}^{\prime}(0)=\lim _{x \rightarrow 0+} \frac{W^{(q)}(x)}{x} .
$$

We remark that $W_{+}^{\prime}(0)$ is finite if and only if either $X$ has a nontrivial Brownian component, or the Lévy measure $\Pi$ has a finite total mass. Therefore, $W(0)=0$ and $W_{+}^{\prime}(0)<\infty$ if and only if $X$ has a nontrivial Brownian component, and in this case $W_{+}^{\prime}(0)=2 / \sigma^{2}$; see the proof of Lemma 4 of [18]. Integrating by parts on its Laplace transform we can also show that

$$
\lim _{x \rightarrow 0+} x W^{(q)^{\prime}}(x)=0 .
$$

The scale function $W^{(q)}$ is related to the excursion measure. Write $\mathcal{E}^{(a)}$ for the set of generic excursions with height at least $a$. Write $\hat{n}$, defined on $\bigcup_{a>0} \varepsilon^{(a)}$, for the excursion measure of $\hat{Y}$. By Theorem VII.8 of [3] we have

$$
\hat{n}\left(\mathcal{E}^{(a)}\right)=\frac{W^{\prime}(a)}{W(a)} .
$$

More relationships like this can be found in [7].

Throughout this paper, we assume that either the paths of $X$ are of unbounded variation or that the Lévy measure $\Pi$ for $X$ is absolutely continuous with respect to the Lebesgue measure. As a result, $W^{(q)}$ is continuously differentiable over the interval $(0, \infty)$.

\section{The conditional exit problem}

In this section we introduce several preliminary results concerning the exit problem for the spectrally negative Lévy process conditioning on exiting from below. They will settle a technical issue arising from the proofs in Section 4 and Section 5. 
For any $a, b \geq 0$, set

$$
T(-a, b):=\inf \left\{t \geq 0: X_{t} \notin(-a, b)\right\} .
$$

Given $\varepsilon>0$ and $a>0$, let $X_{\varepsilon}(\cdot):=X\left(\cdot \wedge T_{\varepsilon}\right)$ denote the process obtained by stopping $X$ at time $T_{\varepsilon}:=T(-a, \varepsilon)$. Let $\left(\mathcal{F}_{t}^{\varepsilon}\right)$ denote the filtration generated by process $X_{\varepsilon}$.

For $x \in(-\infty, \varepsilon)$, let $\mathrm{P}_{\varepsilon}^{x}$ denote the probability law for $X_{\varepsilon}$ starting at $x$ and conditioning on the event such that $\left\{X_{\varepsilon}\left(T_{\varepsilon}\right)=X\left(T_{\varepsilon}\right) \leq-a\right\}$, i.e. for any $x \in(-a, \varepsilon)$ and any $A \in \mathcal{F}_{t}^{\varepsilon}$, by (2.2) and the Markov property, we define

$$
\begin{aligned}
\mathrm{P}_{\varepsilon}^{x}(A) & :=\mathrm{P}^{x}\left\{A \mid X\left(T_{\varepsilon} \leq-a\right\}\right. \\
& =\frac{W(a+\varepsilon)}{W(a+\varepsilon)-W(a+x)} \mathrm{E}^{x}\left[\left(\left(1-\frac{W\left(a+X_{t}\right)}{W(a+\varepsilon)}\right) \mathbf{1}_{\left\{t<T_{\varepsilon}\right\}}+\mathbf{1}_{\left\{t \geq T_{\varepsilon}, X\left(T_{\varepsilon}\right) \leq-a\right\}}\right) \mathbf{1}_{A}\right]
\end{aligned}
$$

and, for any $x \in(-\infty,-a]$, we define

$$
\mathrm{P}_{\varepsilon}^{x}\left\{X_{\varepsilon}(t)=x \text { for all } t \geq 0\right\}=1 .
$$

Set $\mathrm{P}_{\varepsilon}:=\mathrm{P}_{\varepsilon}^{0}$. Then $\left(\mathrm{P}_{\varepsilon}^{x}, x<\varepsilon\right)$ is the probability law of $X_{\varepsilon}$ conditional on the event that $X_{\varepsilon}$ exits the interval $(-a, \varepsilon)$ at $-a$. Note that $X_{\varepsilon}$ is a Markov process under $\left(\mathrm{P}_{\varepsilon}^{x}, x<\varepsilon\right)$. See [3, Section VII.3] and [15] for similar conditional Lévy processes.

The following lemma concerns the exit problem for the conditional process.

Lemma 3.1. Given $c$ and $d$ such that $-a \leq c<x<d<\varepsilon$, we have

$$
\mathrm{P}_{\varepsilon}^{x}\left\{X_{\varepsilon}(T(c, d))=d\right\}=\frac{(W(a+\varepsilon)-W(a+d)) W(x-c)}{(W(a+\varepsilon)-W(a+x)) W(d-c)}
$$

and

$$
\begin{aligned}
\mathrm{E}_{\varepsilon}^{x}\left[\mathrm{e}^{-q T(c, \varepsilon)}\right] & \\
=1-q \int_{c}^{\varepsilon} & \mathrm{d} y \frac{W(a+\varepsilon)-W(a+y)}{W(a+\varepsilon)-W(a+x)} \\
& \times\left(\frac{W^{(q)}(x-c) W^{(q)}(\varepsilon-y)}{W^{(q)}(\varepsilon-c)}-W^{(q)}(x-y) \mathbf{1}_{\{y \leq x\}}\right) .
\end{aligned}
$$

Proof. Equation (3.1) just follows from the definition of $\mathrm{P}_{\varepsilon}^{x}$ and (2.2).

Observe that on the one hand we have

$$
\begin{aligned}
\mathrm{E}_{\varepsilon}^{x}[ & \left.\int_{0}^{T(c, \varepsilon)} \mathrm{d} t \mathrm{e}^{-q t}\right] \\
& =\int_{0}^{\infty} \mathrm{d} t \mathrm{e}^{-q t} \mathrm{P}_{\varepsilon}^{x}\{t<T(c, \varepsilon)\} \\
& =\int_{0}^{\infty} \mathrm{d} t \mathrm{e}^{-q t} \int_{b}^{\varepsilon} \frac{W(a+\varepsilon)-W(a+y)}{W(a+\varepsilon)-W(a+x)} \mathrm{P}^{x}\left\{X_{t} \in \mathrm{d} y, t<T(c, \varepsilon)\right\} \\
& =\int_{c}^{\varepsilon} \mathrm{d} y \frac{W(a+\varepsilon)-W(a+y)}{W(a+\varepsilon)-W(a+x)}\left(\frac{W^{(q)}(x-c) W^{(q)}(\varepsilon-y)}{W^{(q)}(\varepsilon-c)}-W^{(q)}(x-y) \mathbf{1}_{\{y \leq x\}}\right),
\end{aligned}
$$


where we need Theorem 1 of [5] for the last equality, and on the other hand we have

$$
\mathrm{E}_{\varepsilon}^{x}\left[\int_{0}^{T(c, \varepsilon)} \mathrm{d} t \mathrm{e}^{-q t}\right]=\frac{1}{q}\left(1-\mathrm{E}_{\varepsilon}^{x}\left[\mathrm{e}^{-q T(c, \varepsilon)}\right]\right) .
$$

Then the Laplace transform, (3.2), readily follows from (3.3) and (3.4).

Remark 3.1. By Lemma 3.1 we can verify the following facts:

$$
\lim _{\varepsilon \rightarrow 0+} \mathrm{P}_{\varepsilon}^{x}\left\{X_{\varepsilon}(T(-a, 0)) \leq-a\right\}=1 \quad \text { for all } x \in(-a, 0)
$$

and

$$
\lim _{c \rightarrow 0-} \lim _{\varepsilon \rightarrow 0+} \mathrm{E}_{\varepsilon}\left[\mathrm{e}^{-q T(c, \varepsilon)} \mathbf{1}_{\left\{X_{\varepsilon}(T(c, \varepsilon)) \leq c\right\}}\right]=1 .
$$

Moreover, (3.5) holds uniformly for $x$ bounded away from 0 .

Set

$$
\varsigma_{\varepsilon}:=\sup \{t<T(-a, \varepsilon): X(t) \geq 0\}=\sup \left\{t<T(-a, \varepsilon): X_{\varepsilon}(t) \geq 0\right\},
$$

with the convention $\sup \varnothing:=\infty$. Then, for any $c \in(-a, 0)$, we see that $\varsigma_{\varepsilon} \leq T(c, \varepsilon)$ on the event

$$
\left\{X_{\varepsilon}(T(c, \varepsilon)) \leq c, X_{\varepsilon} \circ \theta_{T(c, \varepsilon)}(T(-a, 0)) \leq-a\right\},
$$

where $\theta_{t}$ stands for the shift operator, i.e. $X_{s} \circ \theta_{t}=X_{t+s}$. Consequently, we find that

$$
\begin{aligned}
\mathrm{E}_{\varepsilon}\left[\exp \left(-q \zeta_{\varepsilon}\right)\right] \geq & \mathrm{E}_{\varepsilon}\left[\mathrm{e}^{-q T(c, \varepsilon)} \mathbf{1}_{\left\{X_{\varepsilon}(T(c, \varepsilon))=c\right\}}\right] \mathrm{P}_{\varepsilon}^{c}\left\{X_{\varepsilon}(T(-a, 0)) \leq-a\right\} \\
& +\int_{-\infty}^{c-} \mathrm{E}_{\varepsilon}\left[\mathrm{e}^{-q T(c, \varepsilon)} \mathbf{1}_{\left\{X_{\varepsilon}(T(c, \varepsilon)) \in \mathrm{d} x\right\}}\right] \mathrm{P}_{\varepsilon}^{x}\left\{X_{\varepsilon}(T(-a, 0)) \leq-a\right\} .
\end{aligned}
$$

We can first let $\varepsilon \rightarrow 0+$ in (3.7) and then let $c \rightarrow 0+$ in (3.7). Now it follows, from (3.5) and (3.6), that

$$
\lim _{\varepsilon \rightarrow 0+} \mathrm{E}\left[\exp \left(-q \varsigma_{\varepsilon}\right) \mid X(T(-a, \varepsilon)) \leq-a\right]=\lim _{\varepsilon \rightarrow 0+} \mathrm{E}_{\varepsilon}\left[\exp \left(-q \varsigma_{\varepsilon}\right)\right]=1 .
$$

Remark 3.2. We can also show that (3.8) holds if $T(-a, \varepsilon)$ is replaced by $T(\varepsilon-a, \varepsilon)$, and $\varsigma_{\varepsilon}$ is replaced by

$$
\varsigma_{\varepsilon}^{\prime}:=\sup \{t<T(\varepsilon-a, \varepsilon): X(t) \geq 0\} .
$$

Remark 3.3. Similarly, for $b>0$ and $\varsigma_{\varepsilon}^{\prime \prime}:=\sup \{t<T(-\varepsilon, b): X(t) \leq 0\}$, we can show that

$$
\lim _{\varepsilon \rightarrow 0+} \mathrm{E}\left[\exp \left(-q \varsigma_{\varepsilon}^{\prime \prime}\right) \mid X(T(-\varepsilon, b))=b\right]=1 .
$$

\section{Lévy processes reflected at the supremum}

We are ready to introduce one of the main results in the paper. For $r \leq 0$, write $\bar{\Pi}(r):=$ $\Pi((-\infty, r])$. For $z \geq a>0$, write

$$
\begin{aligned}
H_{\alpha}(z):= & W^{(\alpha)}(0) W^{(\alpha)}(a) \bar{\Pi}(-z)+W^{(\alpha)}(a) \int_{0}^{a} \mathrm{~d} y W^{(\alpha)^{\prime}}(y) \bar{\Pi}(y-z) \\
& -W^{(\alpha)^{\prime}}(a) \int_{0}^{a} \mathrm{~d} y W^{(\alpha)}(y) \bar{\Pi}(y-z)
\end{aligned}
$$


and

$$
V_{\alpha}(a):=\alpha \bar{W}^{(\alpha)}(a) W^{(\alpha) \prime}(a)-\alpha W^{(\alpha)}(a)^{2}+W^{(\alpha) \prime}(a) .
$$

Note that $H_{\alpha}$ is continuous under the assumption given at the end of Section 2.

Recall from the definition in Section 1 that $\tau_{-}(a)$ denotes the starting time (in the usual time scale) of the first excursion (from 0) of $\hat{Y}$ with height at least $a$. Observe that $X_{\tau-(u)}=$ $S_{\tau-(u)}=S_{\tau(u)}$.

Our next result concerns the joint distribution of ( $\left.\tau(a), S_{\tau(a)}, \tau_{-}(a), \hat{Y}_{\tau(a)}\right)$. Note that when $\hat{Y}$ exceeds a certain level, it could either reach the level or overshoot it. We then consider the two cases separately. It partially generalizes Theorem 1 and Theorem 2 of [19]. Our approach is different from that in [19].

Theorem 4.1. For any $z>a>0$ and any $\alpha, \beta, \gamma>0$, we have

$$
\mathrm{E}\left[\exp \left(-\alpha \tau(a)-\beta S_{\tau(a)}-\gamma \tau_{-}(a)\right) \mathbf{1}_{\left\{\hat{Y}_{\tau(a) \geq z\}}\right.}\right]=\frac{W^{(\alpha+\gamma)}(a) H_{\alpha}(z)}{W^{(\alpha)}(a)\left(W^{(\alpha+\gamma) \prime}(a)+\beta W^{(\alpha+\gamma)}(a)\right)}
$$

and

$$
\mathrm{E}\left[\exp \left(-\alpha \tau(a)-\beta S_{\tau(a)}-\gamma \tau_{-}(a)\right) \mathbf{1}_{\left\{\hat{Y}_{\tau(a)}=a\right\}}\right]=\frac{W^{(\alpha+\gamma)}(a)\left(V_{\alpha}(a)-H_{\alpha}(a)\right)}{W^{(\alpha)}(a)\left(W^{(\alpha+\gamma)^{\prime}}(a)+\beta W^{(\alpha+\gamma)}(a)\right)} .
$$

Proof. For $z>a>\varepsilon>0$, set

$$
\Psi:=\mathrm{E}\left[\exp \left(-\alpha \tau(a)-\beta S_{\tau(a)}-\gamma \tau_{-}(a)\right) \mathbf{1}_{\left\{\tau(a)<\infty, \hat{Y}_{\tau(a)} \geq z\right\}}\right] .
$$

Recall that $\zeta_{\varepsilon}^{\prime}$ is defined in (3.9). By distinguishing whether $S_{\tau(a)} \geq \varepsilon$ or $S_{\tau(a)}<\varepsilon$ and applying the strong Markov property, we readily see that, for small enough $\varepsilon$,

$$
\begin{aligned}
\Psi \leq & \mathrm{E}\left[\exp (-(\alpha+\gamma) T(-a, \varepsilon)-\beta \varepsilon) \mathbf{1}_{\left\{X_{T(-a, \varepsilon)}=\varepsilon\right\}}\right] \Psi \\
& +\mathrm{E}\left[\exp (-\alpha T(-a, \varepsilon)) \mathbf{1}_{\left\{X_{T(-a, \varepsilon)} \leq \varepsilon-z\right\}}\right]
\end{aligned}
$$

and

$$
\begin{aligned}
\Psi \geq & \mathrm{E}\left[\exp (-(\alpha+\gamma) T(\varepsilon-a, \varepsilon)-\beta \varepsilon) \mathbf{1}_{\left\{X_{T(\varepsilon-a, \varepsilon)}=\varepsilon\right\}}\right] \Psi \\
& +\mathrm{E}\left[\exp \left(-\alpha T(\varepsilon-a, \varepsilon)-\gamma \varsigma_{\varepsilon}^{\prime}-\beta \varepsilon\right) \mathbf{1}_{\left\{X_{T(\varepsilon-a, \varepsilon)} \leq-z\right\}}\right] .
\end{aligned}
$$

To simplify the expressions, we set $\lambda=\alpha+\gamma$ throughout the proof. Solving (4.3) and (4.4) for $\Psi$, we respectively find that

$$
\Psi \leq \frac{\mathrm{E}\left[\mathrm{e}^{-\alpha T(-a, \varepsilon)} \mathbf{1}_{\left\{X_{T(-a, \varepsilon)} \leq \varepsilon-z\right\}}\right]}{1-\mathrm{E}\left[\exp (-\lambda T(-a, \varepsilon)-\beta \varepsilon) \mathbf{1}_{\left\{X_{T(-a, \varepsilon)}=\varepsilon\right\}}\right]}
$$

and

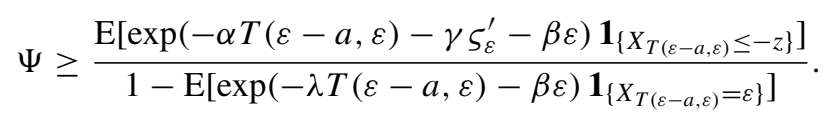

We want to show that

$$
\lim _{\varepsilon \rightarrow 0} \frac{\mathrm{E}\left[\mathrm{e}^{-\alpha T(-a, \varepsilon)} \mathbf{1}_{\left\{X_{T(-a, \varepsilon)} \leq \varepsilon-z\right\}}\right]}{1-\mathrm{E}\left[\exp (-\lambda T(-a, \varepsilon)-\beta \varepsilon) \mathbf{1}_{\left\{X_{T(-a, \varepsilon)}=\varepsilon\right\}}\right]}=\frac{W^{(\lambda)}(a) H_{\alpha}(z)}{W^{(\alpha)}(a)\left(W^{(\lambda) \prime}(a)+\beta W^{(\lambda)}(a)\right)} .
$$


Firstly, by (2.2) we find that

$$
\begin{aligned}
\lim _{\varepsilon \rightarrow 0} & \frac{1}{\varepsilon}\left(1-\mathrm{E}\left[\exp (-\lambda T(-a, \varepsilon)-\beta \varepsilon) \mathbf{1}_{\left\{X_{T(-a, \varepsilon)}=\varepsilon\right\}}\right]\right) \\
& =\lim _{\varepsilon \rightarrow 0} \frac{1}{\varepsilon}\left(1-\frac{\mathrm{e}^{-\beta \varepsilon} W^{(\lambda)}(a)}{W^{(\lambda)}(a+\varepsilon)}\right) \\
& =\frac{W^{(\lambda) \prime}(a)}{W^{(\lambda)}(a)}+\beta .
\end{aligned}
$$

Secondly, by (2.6) we have

$$
\begin{aligned}
& \lim _{\varepsilon \rightarrow 0} \frac{1}{\varepsilon} \mathrm{E}\left[\mathrm{e}^{-\alpha T(-a, \varepsilon)} \mathbf{1}_{\left\{X_{T(-a, \varepsilon)} \leq \varepsilon-z\right\}}\right] \\
&=\lim _{\varepsilon \rightarrow 0} \frac{1}{\varepsilon} \int_{-a}^{\varepsilon} \mathrm{d} y \tilde{W}^{(q)}(a, \varepsilon, y) \bar{\Pi}(\varepsilon-y-z) \\
&=W^{(\alpha)}(0) \bar{\Pi}(-z)+\lim _{\varepsilon \rightarrow 0} \frac{1}{\varepsilon} \int_{0}^{a} \mathrm{~d} y\left(\frac{W^{(\alpha)}(a) W^{(\alpha)}(y+\varepsilon)}{W^{(\alpha)}(a+\varepsilon)}-W^{(\alpha)}(y)\right) \bar{\Pi}(y+\varepsilon-z) \\
&=W^{(\alpha)}(0) \bar{\Pi}(-z)+\lim _{\varepsilon \rightarrow 0} \frac{1}{\varepsilon} \int_{0}^{a} \mathrm{~d} y\left(W^{(\alpha)}(y+\varepsilon)-W^{(\alpha)}(y)\right) \bar{\Pi}(y+\varepsilon-z) \\
& \\
& \quad+\lim _{\varepsilon \rightarrow 0} \frac{1}{\varepsilon} \int_{0}^{a} \mathrm{~d} y\left(\frac{W^{(\alpha)}(a) W^{(\alpha)}(y+\varepsilon)}{W^{(\alpha)}(a+\varepsilon)}-W^{(\alpha)}(y+\varepsilon)\right) \bar{\Pi}(y+\varepsilon-z) \\
&= W^{(\alpha)}(0) \bar{\Pi}(-z)+\int_{0}^{a} \mathrm{~d} y W^{(\alpha) \prime}(y) \bar{\Pi}(y-z)-\frac{W^{(\alpha) \prime}(a)}{W^{(\alpha)}(a)} \int_{0}^{a} \mathrm{~d} y W^{(\alpha)}(y) \bar{\Pi}(y-z) \\
&= \frac{H_{\alpha}(z)}{W^{(\alpha)}(a)} .
\end{aligned}
$$

Then (4.6) follows readily.

Furthermore, we can show that (4.6) also holds if $T(-a, \varepsilon)$ is replaced by $T(\varepsilon-a, \varepsilon)$. From (3.8), observe that

$$
\begin{aligned}
& \lim _{\varepsilon \rightarrow 0} \frac{\mathrm{E}\left[\exp \left(-\alpha T(\varepsilon-a, \varepsilon)-\gamma \zeta_{\varepsilon}^{\prime}\right) \mathbf{1}_{\left\{X_{T(\varepsilon-a, \varepsilon)} \leq-z\right\}}\right]}{\mathrm{E}\left[\exp (-\alpha T(\varepsilon-a, \varepsilon)) \mathbf{1}_{\left\{X_{T(\varepsilon-a, \varepsilon)} \leq-z\right\}}\right]} \\
& \quad=\lim _{\varepsilon \rightarrow 0} \frac{\mathrm{E}\left[\exp \left(-\alpha T(\varepsilon-a, \varepsilon)-\gamma \zeta_{\varepsilon}^{\prime}\right) \mid X_{T(\varepsilon-a, \varepsilon)} \leq-z\right]}{\mathrm{E}\left[\exp (-\alpha T(\varepsilon-a, \varepsilon)) \mid X_{T(\varepsilon-a, \varepsilon)} \leq-z\right]} \\
& \quad=1 .
\end{aligned}
$$

Therefore,

$$
\begin{gathered}
\lim _{\varepsilon \rightarrow 0} \frac{\mathrm{E}\left[\exp \left(-\alpha T(\varepsilon-a, \varepsilon)-\gamma \varsigma_{\varepsilon}^{\prime}-\beta \varepsilon\right) \mathbf{1}_{\left\{X_{T(\varepsilon-a, \varepsilon)} \leq-z\right\}}\right]}{1-\mathrm{E}\left[\exp (-\lambda T(\varepsilon-a, \varepsilon)-\beta \varepsilon) \mathbf{1}_{\left\{X_{T(\varepsilon-a, \varepsilon)}=\varepsilon\right\}}\right]} \\
=\frac{W^{(\lambda)}(a) H_{\alpha}(z)}{W^{(\alpha)}(a)\left(W^{(\lambda) \prime}(a)+\beta W^{(\lambda)}(a)\right)} .
\end{gathered}
$$

We have thus proved (4.1). 
By arguments similar to those used in (4.3)-(4.5), we obtain

$$
\begin{aligned}
& \mathrm{E}\left[\exp \left(-\alpha \tau(a)-\beta S_{\tau(a)}-\gamma \tau_{-}(a)\right)\right] \\
& \quad=\lim _{\varepsilon \rightarrow 0} \frac{\mathrm{E}\left[\mathrm{e}^{-\alpha T(-a, \varepsilon)} \mathbf{1}_{\left\{X_{T(-a, \varepsilon)} \leq-a\right\}}\right]}{1-\mathrm{E}\left[\exp (-\lambda T(-a, \varepsilon)-\beta \varepsilon) \mathbf{1}_{\left\{X_{T(-a, \varepsilon)}=\varepsilon\right\}}\right]} .
\end{aligned}
$$

By (2.3) we obtain

$$
\begin{aligned}
\lim _{\varepsilon \rightarrow 0} \frac{1}{\varepsilon} \mathrm{E}\left[\mathrm{e}^{-\alpha T(-a, \varepsilon)} \mathbf{1}_{\left\{X_{T(-a, \varepsilon)} \leq-a\right\}}\right] & =\lim _{\varepsilon \rightarrow 0} \frac{1}{\varepsilon}\left(\hat{W}^{(\alpha)}(a, \varepsilon)-\frac{W^{(\alpha)}(a)}{W^{(\alpha)}(\varepsilon+a)}\right) \\
& =\frac{V_{\alpha}(a)}{W^{(\alpha)}(a)} ;
\end{aligned}
$$

thus, (4.2) follows from a combination of (4.7), (4.8), (4.9), and (4.10).

Remark 4.1. Given $x \in(0, a)$, by (2.2), (2.6), and (2.7), we can easily find expressions for (4.1) and (4.2) under $\mathrm{E}^{-x}$ by distinguishing whether $X$ first exits the interval $(-a, 0)$ from its upper boundary or from its lower boundary.

Remark 4.2. Combining (4.1) and (4.2), we have

$$
\mathrm{E}\left[\exp \left(-\alpha \tau(a)-\beta S_{\tau(a)}-\gamma \tau_{-}(a)\right)\right]=\frac{W^{(\alpha+\gamma)}(a) V_{\alpha}(a)}{W^{(\alpha)}(a)\left(W^{(\alpha+\gamma) \prime}(a)+\beta W^{(\alpha+\gamma)}(a)\right)} .
$$

By (4.11) we further have

$$
\begin{gathered}
\mathrm{E}\left[\mathrm{e}^{-\gamma \tau(a)}\right]=1+\alpha \bar{W}^{(\alpha)}(a)-\frac{\alpha W^{(\alpha)}(a)^{2}}{W^{(\alpha)^{\prime}}}, \\
\mathrm{E}\left[\mathrm{e}^{-\gamma \tau_{-}(a)}\right]=\frac{W^{\prime}(a) W^{(\gamma)}(a)}{W(a) W^{(\gamma)^{\prime}}(a)},
\end{gathered}
$$

and

$$
\mathrm{E}\left[\exp \left(-\beta S_{\tau(a)}\right)\right]=\frac{W^{\prime}(a)}{W^{\prime}(a)+\beta W(a)}
$$

In particular, $S_{\tau(a)}$ follows an exponential distribution with mean $W(a) / W^{\prime}(a)$; for an interpretation of the ratio $W^{(\gamma)}(a) / W^{(\gamma) \prime}(a)$, see [7, Lemma 2] .

Arguments similar to those in Theorem 4.1 also lead to a result on the inverse local time $L^{-1}$.

Proposition 4.1. For any $x, a>0$, we have

$$
\mathrm{E}\left[\exp \left(-\alpha L^{-1}(x)\right) \mathbf{1}_{\left\{L^{-1}(x)<\tau(a)\right\}}\right]=\exp \left(-\frac{W^{(\alpha) \prime}(a) x}{W^{(\alpha)}(a)}\right) .
$$

Proof. Define

$$
g(x):=\mathrm{E}\left[\exp \left(-\alpha L^{-1}(x)\right) \mathbf{1}_{\left\{L^{-1}(x)<\tau(a)\right\}}\right] .
$$


Applying the strong Markov properties at exit times $T(\varepsilon-a, \varepsilon)$ and $T(-a, \varepsilon)$, respectively, we have

$$
\begin{aligned}
\mathrm{E}\left[\mathrm{e}^{-\alpha T(\varepsilon-a, \varepsilon)} \mathbf{1}_{\{X(T(\varepsilon-a, \varepsilon))=\varepsilon\}}\right] g(x-\varepsilon) \\
\quad \leq g(x) \\
\quad \leq \mathrm{E}\left[\mathrm{e}^{-\alpha T(-a, \varepsilon)} \mathbf{1}_{\{X(T(-a, \varepsilon))=\varepsilon\}}\right] g(x-\varepsilon) .
\end{aligned}
$$

Letting $\varepsilon$ tend to 0 , then $g$ solves the following differential equation

$$
\begin{aligned}
g^{\prime}(x) & =\frac{\lim _{\varepsilon \rightarrow 0} g(x-\varepsilon)\left(\mathrm{E}\left[\mathrm{e}^{-\alpha T(\varepsilon-a, \varepsilon)} \mathbf{1}_{\{X(T(\varepsilon-a, \varepsilon))=\varepsilon\}}\right]-1\right)}{\varepsilon} \\
& =-\frac{g(x) W^{(\alpha) \prime}(a) x}{W^{(\alpha)}(a)} .
\end{aligned}
$$

So, (4.13) follows.

Remark 4.3. It is easy to see that (4.13) also leads to (2.11).

Remark 4.4. Let $a$ tend to $\infty$ in (4.13). Comparing with (2.1) we find that

$$
\lim _{a \rightarrow \infty} \frac{W^{(\alpha) \prime}(a)}{W^{(\alpha)}(a)}=\Phi(\alpha) .
$$

We have thus recovered Lemma 1 of [1] and Lemma 2 of [19].

Remark 4.5. We can also recover the distribution for $S_{\tau(a)}$ by letting $\alpha=0$ in (4.13).

Remark 4.6. If $X$ has a downward drift, then 0 is transient for $\hat{Y}$. Let $\tau_{-}(\infty)$ denote the arrival time of the unique excursion with infinite lifetime. Note that $\tau_{-}(\infty)$ is also the last time when $X$ reaches its overall supremum $S_{\tau_{-}(\infty)}$. It follows, from (4.11) and (4.14), that

$$
\begin{aligned}
\mathrm{E}\left[\exp \left(-\beta S_{\tau_{-}(\infty)}-\gamma \tau_{-}(\infty)\right)\right] & =\lim _{a \rightarrow \infty} \frac{W^{\prime}(a) W^{(\gamma)}(a)}{W(a)\left(W^{(\gamma)^{\prime}}(a)+\beta W^{(\gamma)}(a)\right)} \\
& =\frac{\Phi(0)}{\Phi(\gamma)+\beta} .
\end{aligned}
$$

Define

$$
l(a):=\inf \left\{t>0: \hat{Y}_{\tau_{-}(a)+t}=0\right\},
$$

with the convention inf $\varnothing:=\infty$. Then $l(a)$ denotes the length of the first excursion with height at least $a$. Modifying the arguments used in Theorem 4.1 leads to the Laplace transform for $\left(\tau_{-}(a), X_{\tau_{-}(a)}, l(a)\right)$.

Theorem 4.2. For any $a>0$ and any $\alpha, \beta, \gamma>0$, we have

$$
\begin{aligned}
\mathrm{E}\left[\exp \left(-\alpha \tau_{-}(a)-\beta X_{\tau_{-}(a)}-\gamma l(a)\right)\right] & \\
= & \frac{W^{(\alpha)}(a)}{\left(W^{(\alpha) \prime}(a)+\beta W^{(\alpha)}(a)\right) W^{(\gamma)}(a)} \\
& \times\left(\left(V_{\gamma}(a)-H_{\gamma}(a)\right) \mathrm{e}^{-a \Phi(\gamma)}-\int_{a+}^{\infty} \mathrm{e}^{-x \Phi(\gamma)} \mathrm{d} H_{\gamma}(x)\right) .
\end{aligned}
$$


Proof. Considering how process $X$ exits the intervals $(-a, \varepsilon)$ or $(\varepsilon-a, \varepsilon)$, similar to the proof of Theorem 4.1, we can show that, for $T:=T_{(-a, \varepsilon)}$,

$$
\begin{aligned}
\mathrm{E}\left[\exp \left(-\alpha \tau_{-}(a)-\beta X_{\tau_{-}(a)}-\gamma l(a)\right)\right]= & \lim _{\varepsilon \rightarrow 0} \frac{\mathrm{E}\left[\mathrm{e}^{-\gamma l(a)} \mathbf{1}_{\left\{X_{T} \leq-a\right\}}\right]}{1-\mathrm{E}\left[\mathrm{e}^{-\alpha T-\beta \varepsilon} \mathbf{1}_{\left\{X_{T}=\varepsilon\right\}}\right]} \\
= & \lim _{\varepsilon \rightarrow 0} \frac{\mathrm{E}\left[\mathrm{e}^{-\gamma T} \mathbf{1}_{\left\{X_{T}=-a\right\}}\right] \mathrm{e}^{-a \Phi(\gamma)}}{1-\mathrm{e}^{-\beta \varepsilon} \mathrm{E}\left[\mathrm{e}^{-\alpha T} \mathbf{1}_{\left\{X_{T}=\varepsilon\right\}}\right]} \\
& +\lim _{\varepsilon \rightarrow 0} \frac{\int_{-\infty}^{-a-} \mathrm{E}\left[\mathrm{e}^{-\gamma T} \mathbf{1}_{\left\{X_{T} \in \mathrm{d} x\right\}}\right] \mathrm{e}^{x \Phi(\gamma)}}{1-\mathrm{e}^{-\beta \varepsilon} \mathrm{E}\left[\mathrm{e}^{-\alpha T} \mathbf{1}_{\left\{X_{T}=\varepsilon\right\}}\right]},
\end{aligned}
$$

where (2.1) was used in the last equation.

So, (4.16) follows from combining (4.7), (4.8), and (4.10).

Note that 0 is recurrent for $\hat{Y}$ if either $X$ is recurrent or $\lim _{t \rightarrow \infty} X_{t}=\infty$. In this case the excursion process for $\hat{Y}$ is a Poisson point process.

Set $\bar{Y}_{t}:=\sup _{0 \leq s \leq t} \hat{Y}_{s}$. We can easily find the distribution for $\bar{Y}_{\tau_{-}(a)}$.

Proposition 4.2. Suppose that 0 is recurrent for $\hat{Y}$, then, for any $0<y<a$, we have

$$
\mathrm{P}\left\{\bar{Y}_{\tau_{-}(a)}<y\right\}=\frac{W(y) W^{\prime}(a)}{W^{\prime}(y) W(a)} .
$$

Proof. Consider the Poisson point process of excursions in $\mathcal{E}^{(y)}$. Note that $\left\{\bar{Y}_{\tau_{-}(a)}<y\right\}$ is also the event that the first arrived excursion of height at least $y$ is indeed an excursion of height at least $a$. Then it follows, from (2.11), that

$$
\mathrm{P}\left\{\bar{Y}_{\tau_{-}(a)}<y\right\}=\frac{\hat{n}\left(\mathscr{E}^{(a)}\right)}{\hat{n}\left(\mathscr{E}^{(y)}\right)}=\frac{W(y) W^{\prime}(a)}{W^{\prime}(y) W(a)} ;
$$

see [3, Proposition 0.5.2].

\section{Lévy processes reflected at the infimum}

Recall from its definition in Section 1 that $\kappa_{-}(b), b>0$, denotes the starting time of the first excursion for $Y$ with height at least $b$. Also note that $X_{\kappa_{-}(b)}=I_{\kappa_{-}(b)}=I_{\kappa(b)}$.

For $\lambda, \beta, b>0$, write

$$
G(\lambda, \beta, b):=\int_{0}^{b} \mathrm{~d} y W^{(\lambda)}(b-y)\left(\bar{\Pi}(-y)-\int_{-\infty}^{0-} \Pi(-y+\mathrm{d} z) \mathrm{e}^{\beta z}\right) .
$$

Note that $G(\lambda, 0, b)=0$.

Our next theorem is parallel to Theorem 4.1. Recall, from Section 2, that $W(0)=0$ and $W_{+}^{\prime}(0)<\infty$ if and only if the Brownian component for $X$ is nontrivial.

Theorem 5.1. For any $b>0$ and any $\alpha, \beta, \gamma>0$,

- if $W(0)>0$, we have

$$
\begin{aligned}
& \mathrm{E}\left[\exp \left(-\alpha \kappa(b)+\beta I_{\kappa(b)}-\gamma \kappa_{-}(b)\right)\right] \\
& \quad=\frac{W^{(\alpha+\gamma)}(b)}{W^{(\alpha)}(b)\left(1+(\alpha+\gamma) \bar{W}^{(\alpha+\gamma)}(b)+G(\alpha+\gamma, \beta, b)\right)}
\end{aligned}
$$


- if $W(0)=0$ and $W_{+}^{\prime}(0)<\infty$, we have

$$
\begin{aligned}
& \mathrm{E}\left[\exp \left(-\alpha \kappa(b)+\beta I_{\kappa(b)}-\gamma \kappa_{-}(b)\right)\right] \\
& \quad=\frac{W^{(\alpha+\gamma)}(b)}{W^{(\alpha)}(b)\left(\beta \sigma^{2} W^{(\alpha+\gamma)}(b) / 2+1+(\alpha+\gamma) \bar{W}^{(\alpha+\gamma)}(b)+G(\alpha+\gamma, \beta, b)\right)}
\end{aligned}
$$

- if $W(0)=0$ and $W_{+}^{\prime}(0)=\infty$, we further suppose that, for any $\lambda \geq 0$, there exits $\delta>0$ such that $W^{(\lambda)}(x) / x$ decreases for $x \in(0, \delta)$, or equivalently, $W^{(\lambda)^{\prime}}(x) x-W^{(\lambda)}(x) \leq 0$ for $x \in(0, \delta)$, then (5.1) holds.

Proof. Similar to the proof of Theorem 4.1, we can show that, for $T:=T(-\varepsilon, b)$,

$$
\begin{aligned}
& \mathrm{E}\left[\exp \left(-\alpha \kappa(b)+\beta I_{\kappa(b)}-\gamma \kappa_{-}(b)\right)\right] \\
& \quad=\lim _{\varepsilon \rightarrow 0} \frac{W^{(\alpha)}(\varepsilon)}{W^{(\alpha)}(b+\varepsilon)\left(1-\mathrm{E}\left[\exp \left(-\lambda T+\beta X_{T}\right) \mathbf{1}_{\left\{X_{T} \leq-\varepsilon\right\}}\right]\right)} .
\end{aligned}
$$

It follows, from (2.3) and (2.6), that

$$
\begin{aligned}
& \mathrm{E}\left[\exp \left(-\lambda T+\beta X_{T}\right) \mathbf{1}_{\left\{X_{T}=-\varepsilon\right\}}\right] \\
& \quad=\mathrm{e}^{-\beta \varepsilon}\left\{\hat{W}^{(\lambda)}(\varepsilon, b)-\frac{W^{(\lambda)}(\varepsilon)}{W^{(\lambda)}(b+\varepsilon)}-\int_{-\varepsilon}^{b} \mathrm{~d} y \tilde{W}^{(\lambda)}(\varepsilon, b, y) \bar{\Pi}(-y-\varepsilon)\right\}
\end{aligned}
$$

and

$$
\mathrm{E}\left[\exp \left(-\lambda T+\beta X_{T}\right) \mathbf{1}_{\left\{X_{T}<-\varepsilon\right\}}\right]=\int_{-\varepsilon}^{b} \mathrm{~d} y \tilde{W}^{(\lambda)}(\varepsilon, b, y) \int_{-\infty}^{-\varepsilon-} \Pi(-y+\mathrm{d} z) \mathrm{e}^{\beta z} .
$$

We then have

$$
\begin{aligned}
1-\mathrm{E}[ & \left.\exp \left(-\lambda T+\beta X_{T}\right) \mathbf{1}_{\left\{X_{T} \leq-\varepsilon\right\}}\right] \\
= & 1-\mathrm{e}^{-\beta \varepsilon}\left\{\hat{W}^{(\lambda)}(\varepsilon, b)-\frac{W^{(\lambda)}(\varepsilon)}{W^{(\lambda)}(b+\varepsilon)}-\int_{0}^{b} \mathrm{~d} y \tilde{W}^{(\lambda)}(\varepsilon, b, y) \bar{\Pi}(-y-\varepsilon)\right\} \\
& -\int_{0}^{b} \mathrm{~d} y \tilde{W}^{(\lambda)}(\varepsilon, b, y) \int_{-\infty}^{-\varepsilon-} \Pi(-y+\mathrm{d} z) \mathrm{e}^{\beta z}+\mathrm{e}^{-\beta \varepsilon} A_{\varepsilon}-\left(1-\mathrm{e}^{-\beta \varepsilon}\right) B_{\varepsilon},
\end{aligned}
$$

where

$$
A_{\varepsilon}:=\int_{-\varepsilon}^{0} \mathrm{~d} y \tilde{W}^{(\lambda)}(\varepsilon, b, y) \int_{-\infty}^{-\varepsilon-} \Pi(-y+\mathrm{d} z)\left(1-\mathrm{e}^{\beta z}\right)
$$

and

$$
B_{\varepsilon}:=\int_{-\varepsilon}^{0} \mathrm{~d} y \tilde{W}^{(\lambda)}(\varepsilon, b, y) \int_{-\infty}^{-\varepsilon-} \Pi(-y+\mathrm{d} z) \mathrm{e}^{\beta z} .
$$

If $W(0)=0$ then $W^{(\alpha)}(0)=W(0)=0$, and condition (1.1) implies that

$$
\int_{-\infty}^{0} \mathrm{~d} x 1 \wedge(-x) \bar{\Pi}(x)<\infty
$$


For $W_{+}^{\prime}(0)<\infty$, by integration by parts we obtain

$$
\begin{aligned}
\lim _{\varepsilon \rightarrow 0} \frac{A_{\varepsilon}}{\varepsilon}= & \lim _{\varepsilon \rightarrow 0} \frac{1}{\varepsilon} \int_{-\varepsilon}^{0} \mathrm{~d} y\left(\frac{W^{(\lambda)}(\varepsilon) W^{(\lambda)}(b-y)}{W^{(\lambda)}(b+\varepsilon)}-W^{(\lambda)}(-y)\right) \\
& \times\left(\left(1-\mathrm{e}^{-\beta \varepsilon}\right) \bar{\Pi}(-\varepsilon-y)+\int_{-\infty}^{-\varepsilon-} \mathrm{d} z \bar{\Pi}(-y+z) \beta \mathrm{e}^{\beta z}\right) \\
= & \beta \lim _{\varepsilon \rightarrow 0} \int_{-\varepsilon}^{0} \mathrm{~d} y\left(-\frac{W^{(\lambda)}(\varepsilon) W^{(\lambda) \prime}(b)}{W^{(\lambda)}(b+\varepsilon)}+W_{+}^{(\lambda) \prime}(0)\right)(\varepsilon+y) \bar{\Pi}(-\varepsilon-y) \\
& +\lim _{\varepsilon \rightarrow 0} \frac{\beta}{\varepsilon} \int_{-\varepsilon}^{0} \mathrm{~d} y\left(-\frac{W^{(\lambda)}(\varepsilon) W^{(\lambda) \prime}(b)}{W^{(\lambda)}(b+\varepsilon)}+W_{+}^{(\lambda) \prime}(0)\right)(\varepsilon+y) \int_{-\infty}^{-\varepsilon-y} \mathrm{~d} z \bar{\Pi}(z) \\
= & 0,
\end{aligned}
$$

where for the last equality, we have used the fact that $\int_{-\varepsilon}^{0} \mathrm{~d} z(-z) \bar{\Pi}(z) \rightarrow 0$ as $\varepsilon \rightarrow 0$, which follows from (5.3), and the fact that, for any $c>0$,

$$
\begin{aligned}
\lim _{\varepsilon \rightarrow 0} & \frac{1}{\varepsilon} \int_{-\varepsilon}^{0} \mathrm{~d} y(\varepsilon+y) \int_{-\infty}^{-\varepsilon-y} \mathrm{~d} z \bar{\Pi}(z) \\
& \leq \lim _{\varepsilon \rightarrow 0} \frac{1}{\varepsilon} \int_{-\varepsilon}^{0} \mathrm{~d} y(\varepsilon+y) \int_{-\infty}^{-c} \mathrm{~d} z \bar{\Pi}(z)+\lim _{\varepsilon \rightarrow 0} \frac{1}{\varepsilon} \int_{-\varepsilon}^{0} \mathrm{~d} y \int_{-c}^{0} \mathrm{~d} z(-z) \bar{\Pi}(z) .
\end{aligned}
$$

Similarly,

$$
\lim _{\varepsilon \rightarrow 0} \frac{\left(1-\mathrm{e}^{-\beta \varepsilon}\right) B_{\varepsilon}}{\varepsilon}=0 .
$$

For $W_{+}^{\prime}(0)=\infty$, we find that

$$
\begin{aligned}
\lim _{\varepsilon \rightarrow 0} \frac{A_{\varepsilon}}{W^{(\lambda)}(\varepsilon)} & \lim _{\varepsilon \rightarrow 0} \int_{-\varepsilon}^{0} \mathrm{~d} y\left(\frac{W^{(\lambda)}(b-y)}{W^{(\lambda)}(b+\varepsilon)}-\frac{W^{(\lambda)}(-y)}{W^{(\lambda)}(\varepsilon)}\right) \\
& \times\left(\left(1-\mathrm{e}^{-\beta \varepsilon}\right) \bar{\Pi}(-\varepsilon-y)+\int_{-\infty}^{-\varepsilon-} \mathrm{d} z \bar{\Pi}(-y+z) \beta \mathrm{e}^{\beta z}\right) \\
= & \beta \lim _{\varepsilon \rightarrow 0} \int_{-\varepsilon}^{0} \mathrm{~d} y\left(-\frac{\varepsilon W^{(\lambda) \prime}(b)}{W^{(\lambda)}(b)}+\frac{W^{(\lambda)}(\varepsilon)-W^{(\lambda)}(-y)}{(\varepsilon+y) W^{(\lambda)}(\varepsilon) / \varepsilon}\right)(\varepsilon+y) \bar{\Pi}(-\varepsilon-y) \\
& +\lim _{\varepsilon \rightarrow 0} \frac{\beta}{\varepsilon} \int_{-\varepsilon}^{0} \mathrm{~d} y\left(-\frac{\varepsilon W^{(\lambda) \prime}(b)}{W^{(\lambda)}(b+\varepsilon)}+\frac{W^{(\lambda)}(\varepsilon)-W^{(\lambda)}(-y)}{(\varepsilon+y) W^{(\lambda)}(\varepsilon) / \varepsilon}\right)(\varepsilon+y) \int_{-\infty}^{-\varepsilon-y} \mathrm{~d} z \bar{\Pi}(z) \\
= & 0,
\end{aligned}
$$

where for the last equality, we have used the monotonicity assumption on $W^{(\lambda)}(x) / x$ for $x$ close to 0 , i.e. for small enough $\varepsilon$,

$$
0 \leq \frac{W^{(\lambda)}(\varepsilon)-W^{(\lambda)}(-y)}{\varepsilon+y} / \frac{W^{(\lambda)}(\varepsilon)}{\varepsilon} \leq 1 .
$$

Similarly,

$$
\lim _{\varepsilon \rightarrow 0} \frac{\left(1-\mathrm{e}^{-\beta \varepsilon}\right) B_{\varepsilon}}{W^{(\lambda)}(\varepsilon)}=0 .
$$


Consequently, identity (5.2) follows from an argument similar to that used in the proof of Theorem 4.1. Note that identities (2.8) and (2.10), and $W_{+}^{\prime}(0)=2 / \sigma^{2}$ are needed at the end of the argument.

If $W(0)>0$ then $W^{(\alpha)}(0)=W(0)>0$. We also have

$$
\int_{-\infty}^{0} 1 \wedge(-x) \bar{\Pi}(\mathrm{d} x)<\infty
$$

[3, Corollary VII.5], which implies that

$$
\int_{-\infty}^{0} \mathrm{~d} x \Pi(x)<\infty
$$

Identity (5.1) can be obtained similarly.

Remark 5.1. Given $x \in(0, b)$, it follows, from (2.2), (2.6), and (2.7), that with $\lambda=\alpha+\gamma$,

$$
\begin{aligned}
& \mathrm{E}^{x}\left[\exp \left(-\alpha \kappa(b)+\beta I_{\kappa(b)}-\gamma \kappa_{-}(b)\right)\right] \\
&= \frac{W^{(\alpha)}(x)}{W^{(\alpha)}(b)}+\mathrm{E}\left[\exp \left(-\alpha \kappa(b)+\beta I_{\kappa(b)}-\gamma \kappa_{-}(b)\right)\right] \\
& \quad \times\left(\int_{-x}^{b-x} \mathrm{~d} y \tilde{W}^{(q)}(x, b, y) \int_{-\infty}^{-x} \mathrm{e}^{\beta(z+x)} \Pi(-y+\mathrm{d} z)\right. \\
& \quad+\hat{W}^{(\lambda)}(x, b-x)-\frac{W^{(\lambda)}(x)}{W^{(\lambda)}(b)} \\
&\left.\quad-\int_{-x}^{b-x} \mathrm{~d} y \bar{\Pi}(-y-x) \tilde{W}^{(q)}(x, b, y)\right) .
\end{aligned}
$$

Remark 5.2. If 0 is irregular for $(-\infty, 0)$ for $X$, i.e. $W(0)>0$, we can directly obtain the expression for $\mathrm{E}\left[\exp \left(-\alpha \kappa(b)+\beta I_{\kappa(b)}-\gamma \kappa_{-}(b)\right)\right]$ from (5.4) by letting $x \rightarrow 0+$ in (5.4) and then solving the resulting equation.

Remark 5.3. The joint Laplace transform (5.4) yields

$$
\mathrm{E}^{x}\left[\mathrm{e}^{-\alpha \kappa(b)}\right]=\frac{1+\alpha \bar{W}^{(\alpha)}(x)}{1+\alpha \bar{W}^{(\alpha)}(b)},
$$

and we have recovered Proposition 2(i) of [18].

In addition, from (5.4) we obtain

$$
\mathrm{E}^{x}\left[\mathrm{e}^{-\gamma \kappa_{-}(b)}\right]=\frac{W^{(\gamma)}(b)\left(1+\gamma \bar{W}^{(\gamma)}(x)\right)}{W(b)\left(1+\gamma \bar{W}^{(\gamma)}(b)\right)}+\frac{W(x)-W^{(\gamma)}(x)}{W(b)} .
$$

In particular,

$$
\mathrm{E}\left[\mathrm{e}^{-\gamma \kappa_{-}(b)}\right]=\frac{W^{(\gamma)}(b)}{W(b)\left(1+\gamma \bar{W}^{(\gamma)}(b)\right)} .
$$

Remark 5.4. If $X$ is drifted to $+\infty$ then $W$ is necessarily bounded and $\Phi(0)=0$. It follows, from (2.4), that

$$
\lim _{b \rightarrow \infty} W(b)=\frac{1}{\psi^{\prime}(0+)} .
$$


From (2.1) and (2.2), observe that, for $y>0$,

$$
\lim _{b \rightarrow \infty} \frac{W^{(\gamma)}(b-y)}{W^{(\gamma)}(b)}=\mathrm{e}^{-y \Phi(\gamma)} .
$$

Consequently, by Theorem 5.1 and (4.14), we further have

$$
\begin{aligned}
\mathrm{E}\left[\exp \left(\beta I_{\kappa_{-}(\infty)}-\gamma \kappa_{-}(\infty)\right)\right] & =\lim _{b \rightarrow \infty} \mathrm{E}\left[\exp \left(\beta I_{\kappa_{-}(b)}-\gamma \kappa_{-}(b)\right)\right] \\
& =\frac{\psi^{\prime}(0+)}{\beta \sigma^{2} / 2+\gamma / \Phi(\gamma)+G^{*}(\gamma, \beta)}
\end{aligned}
$$

when the Brownian component is nontrivial, and

$$
\mathrm{E}\left[\exp \left(\beta I_{\kappa_{-}(\infty)}-\gamma \kappa_{-}(\infty)\right)\right]=\frac{\psi^{\prime}(0+)}{\gamma / \Phi(\gamma)+G^{*}(\gamma, \beta)}
$$

otherwise (under the condition of Theorem 5.1), where $\kappa_{-}(\infty)$ is the last time when $X$ attains its infimum $I_{\kappa_{-}(\infty)}$ and

$$
G^{*}(\gamma, \beta):=\int_{0}^{\infty} \mathrm{d} y \mathrm{e}^{-y \Phi(\gamma)}\left(\Pi(-y)-\int_{-\infty}^{0-} \Pi(-y+\mathrm{d} z) \mathrm{e}^{\beta z}\right) .
$$

Note that $G^{*}(\gamma, 0)=0$ and $\lim _{\gamma \rightarrow 0+} \gamma / \Phi(\gamma)=\psi^{\prime}(0+)$.

\section{Examples}

For examples we first consider a Brownian motion with drift, i.e. $X_{t}=m t+B_{t}$, where $B_{t}$ is a Brownian motion. Then

$$
\psi(\delta)=\frac{\delta^{2}}{2}+m \delta
$$

and

$$
\Phi(\lambda)=-m+\sqrt{m^{2}+2 \lambda} .
$$

It follows that, for $m \neq 0$,

$$
\begin{aligned}
W^{(\alpha)}(a)=\frac{1}{\sqrt{m^{2}+2 \alpha}} & \left(\exp \left(-a\left(m-\sqrt{m^{2}+2 \alpha}\right)\right)-\exp \left(-a\left(m+\sqrt{m^{2}+2 \alpha}\right)\right)\right), \\
W^{(\alpha) \prime}(a)= & \frac{m+\sqrt{m^{2}+2 \alpha}}{\sqrt{m^{2}+2 \alpha}} \exp \left(-a\left(m+\sqrt{m^{2}+2 \alpha}\right)\right) \\
& -\frac{m-\sqrt{m^{2}+2 \alpha}}{\sqrt{m^{2}+2 \alpha}} \exp \left(-a\left(m-\sqrt{m^{2}+2 \alpha}\right)\right)
\end{aligned}
$$

and

$$
\begin{aligned}
\bar{W}^{(\alpha)}(a)= & \frac{m-\sqrt{m^{2}+2 \alpha}}{2 \alpha \sqrt{m^{2}+2 \alpha}}-\frac{m-\sqrt{m^{2}+2 \alpha}}{2 \alpha \sqrt{m^{2}+2 \alpha}} \exp \left(-a\left(m+\sqrt{m^{2}+2 \alpha}\right)\right) \\
& +\frac{m+\sqrt{m^{2}+2 \alpha}}{2 \alpha \sqrt{m^{2}+2 \alpha}} \exp \left(-a\left(m-\sqrt{m^{2}+2 \alpha}\right)\right)-\frac{m+\sqrt{m^{2}+2 \alpha}}{2 \alpha \sqrt{m^{2}+2 \alpha}} .
\end{aligned}
$$


In addition, for $m=0$,

$$
\begin{gathered}
W^{(\alpha)}(a)=\frac{1}{\sqrt{2 \alpha}}(\exp (a \sqrt{2 \alpha})-\exp (-a \sqrt{2 \alpha})), \\
W^{(\alpha) \prime}(a)=\exp (a \sqrt{2 \alpha})+\exp (-a \sqrt{2 \alpha}),
\end{gathered}
$$

and

$$
\bar{W}^{(\alpha)}(a)=\frac{1}{2 \alpha}(\exp (a \sqrt{2 \alpha})+\exp (-a \sqrt{2 \alpha})-2) .
$$

We can check that

$$
V_{\alpha}(a)=\alpha \bar{W}^{(\alpha)}(a) W^{(\alpha) \prime}(a)-\alpha W^{(\alpha)}(a)^{2}+W^{(\alpha) \prime}(a)=2 \mathrm{e}^{-2 a m} .
$$

Therefore,

$$
\mathrm{E}\left[\exp \left(-\alpha \tau(a)-\beta S_{\tau(a)}-\gamma \tau_{-}(a)\right)\right]=\frac{2 \mathrm{e}^{-2 a m} W^{(\alpha+\gamma)}(a)}{W^{(\alpha)}\left(W^{(\alpha+\gamma) \prime}(a)+\beta W^{(\alpha+\gamma)}(a)\right)},
$$

which is in agreement with Theorem 1 of [25].

By (4.15) we have, for $m<0$,

$$
\mathrm{E}\left[\exp \left(-\beta S_{\tau_{-}(\infty)}-\gamma \tau_{-}(\infty)\right)\right]=\frac{2 m}{m-\sqrt{m^{2}+2 \gamma}-\beta} .
$$

Moreover, by Proposition 4.2 it follows that, for $m>0$,

$$
\begin{aligned}
\mathrm{P}\left\{\bar{Y}_{\tau_{-}(a)}<y\right\} & =\left(\frac{2 m \mathrm{e}^{-2 m a}}{1-\mathrm{e}^{-2 m a}}\right)\left(\frac{1-\mathrm{e}^{-2 m y}}{2 m \mathrm{e}^{-2 m y}}\right) \\
& =\frac{\mathrm{e}^{-2 m a}-\mathrm{e}^{-2 m(a+y)}}{\mathrm{e}^{-2 m y}-\mathrm{e}^{-2 m(a+y)}} .
\end{aligned}
$$

We now consider a completely asymmetric stable process with parameter $1<\theta<2$. Its Laplace exponent is

$$
\psi(\alpha)=\alpha^{\theta}=\frac{\theta(\theta-1)}{\Gamma(2-\theta)} \int_{-\infty}^{0}\left(\mathrm{e}^{\alpha x}-1-\alpha x\right)(-x)^{-\theta-1} \mathrm{~d} x .
$$

Then $\Phi(\lambda)=\lambda^{1 / \theta}$ and

$$
\Pi(\mathrm{d} x)=\frac{\theta(\theta-1)}{\Gamma(2-\theta)}(-x)^{-\theta-1} \mathrm{~d} x, \quad x<0 .
$$

In addition,

$$
W^{(\alpha)}(x)=\theta x^{\theta-1} E_{\theta}^{\prime}\left(\alpha x^{\theta}\right), \quad x \geq 0
$$

where

$$
E_{\theta}(y):=\sum_{n=0}^{\infty} \frac{y^{n}}{\Gamma(1+\theta n)}
$$

denotes the Mittag-Leffler function of parameter $\theta$, and $E_{\theta}^{\prime}$ denotes its derivative. Such an expression for $W^{(\alpha)}$ can be found in [4]. 
We can easily verify the monotonicity assumption on $W^{(\lambda)}(x) / x$ in Theorem 5.1 for the completely asymmetric stable process. Then by (4.12) we have

$$
\mathrm{E}\left[\mathrm{e}^{-\gamma \tau_{-}(a)}\right]=\frac{(\theta-1) E_{\theta}^{\prime}\left(\gamma a^{\theta}\right)}{(\theta-1) E_{\theta}^{\prime}\left(\gamma a^{\theta}\right)+\gamma \theta a^{\theta} E_{\theta}^{\prime \prime}\left(\gamma a^{\theta}\right)} .
$$

Moreover, by (5.6) and (5.7), we have

$$
\mathrm{E}\left[\mathrm{e}^{-\alpha \kappa(b)}\right]=\frac{1}{E_{\theta}\left(\alpha b^{\theta}\right)}
$$

and

$$
\mathrm{E}\left[\mathrm{e}^{-\alpha \kappa_{-}(b)}\right]=\frac{\Gamma(1+\theta) E_{\theta}^{\prime}\left(\alpha b^{\theta}\right)}{E_{\theta}\left(\alpha b^{\theta}\right)} .
$$

We can also derive other explicit, although rather complex expressions for the multidimensional Laplace transforms obtained in Theorem 4.1, Theorem 4.2, and Theorem 5.1. But the details are omitted.

\section{Acknowledgements}

The author thanks Jean Bertoin for pointing out several important references. He also thanks anonymous referees for very helpful comments.

\section{References}

[1] Avram, F., Kyprianou, A. E. and Pistorius, M. R. (2004). Exit problems for spectrally negative Lévy processes and applications to (Canadized) Russian options. Ann. Appl. Prob. 14, 215-238.

[2] Bertoin, J. (1992). An extension of Pitman's theorem for spectrally positive Lévy processes. Ann. Prob. 20, 1464-1483.

[3] Bertoin, J. (1996a). Lévy Processes. Campbridge University Press.

[4] Bertoin, J. (1996b). On the first exit-time of a completely asymmetric stable process from a finite interval. Bull. London Math. Soc. 5, 514-520.

[5] Bertoin, J. (1997). Exponential decay and ergodicity of completely asymmetric Lévy processes in a finite interval. Ann. Appl. Prob. 7, 156-169.

[6] Bingham, N. H. (1975). Fluctuation theory in continuous time. Adv. Appl. Prob. 7, 705-766.

[7] Doney, R. A. (2004). Some excursion calculations for spectrally one-sided Lévy processes. In Séminaire de Probabilités XXXVIII (Lecture Notes Math. 1857), Springer, Berlin, pp. 5-15.

[8] Doney, R. A. (2007). Fluctuation Theory for Lévy Processes (Lecture Notes Math. 1897). Springer, Berlin.

[9] Gerber, H. U. And Shiu, E. S. W. (2004). Optimal dividends: analysis with Brownian motion. N. Amer. Actuarial J. 8, 1-20.

[10] Greenwood, P. and Pitman, J. W. (1980). Fluctuation identities for Lévy processes and splitting at the maximum. Adv. Appl. Prob. 12, 893-902.

[11] Harrison, J. M. (1985). Brownian Motion and Stochastic Flow Systems. John Wiley, New York.

[12] Kyprianou, A. E. (2006). First passage of reflected strictly stable processes. ALEA Latin Amer. J. Prob. Math. Statist. 2, 119-123.

[13] Kyprianou, A. E. (2006). Introductory Lectures on Fluctuations of Lévy Processes with Applications. Springer, Berlin.

[14] Kyprianou, A. E. and Palmowski, Z. (2007). Distributional study of De Finetti's dividend problem for a general Lévy insurance risk process. J. Appl. Prob. 44, 428-443.

[15] Lambert, A. (2000). Completely asymmetric Lévy processes confined in a finite interval. Ann. Inst. H. Poincaré Prob. Statist. 36, 251-274.

[16] Le Gall, J. And Le Jan, Y. (1998). Branching processes in Lévy processes: the exploration process. Ann. Prob. 26, 213-252.

[17] NGuYen-Ngoc, L. And Yor, M. (2004). Some martingales associated to reflected Lévy processes. In Séminaire de Probabilités XXXVIII (Lecture Notes Math. 1857), Springer, Berlin, pp. 42-69. 
[18] Pistorius, M. R. (2004). On exit and ergodicity of the completely asymmetric Lévy process reflected at its infimum. J. Theoret. Prob. 17, 183-220.

[19] Pistorius, M. R. (2007). An excursion theoretical approach to some boundary crossing problems and the Skorokhod embedding for reflected Lévy processes. In Séminaire de Probabilités XL, eds C. Donati-Martin et al., Springer, Berlin, pp. 287-308.

[20] Renaud, J. and Zhou, X. (2007). Distribution of the present value dividend payments in a Lévy risk model. J. Appl. Prob. 44, 420-428.

[21] Revuz, D. ANd Yor, M. (1991). Continuous Martingales and Brownian Motion. Springer, New York.

[22] Rogers, L. C. G. (1984). A new identity for real Lévy processes. Ann. Inst. H. Poincaré Prob. Statist. 20, $12-34$.

[23] Taylor, H. M. (1975). A stopped Brownian motion formula. Ann. Prob. 3, 234-246.

[24] Whitт, W. (2000). An overview of Brownian and non-Brownian FCLTs for the single-server queue. Queueing Systems Theory Appl. 36, 39-70.

[25] Williams, R. J. (1992). Asymptotic variance parameters for the boundary local times of reflected Brownian motion on a compact interval. J. Appl. Prob. 29, 996-1002. 